\title{
Entwicklung eines digitalen Lehr- und Lernarrangements für das deutsche Handwerk
}

\author{
Patrick Spieth $\odot$, Christoph Klos, Tobias Röth $\odot$, Ludger Schmidt, \\ Johannes Funk, Anna Klingauf, Steffi Robak, Moritz Knaut, \\ Maria Klimpel, Lena Heidemann, Friedrich Schüttler und Heiko Gringel
}

\subsection{Digitalisierung des Handwerks: Notwendigkeit einer bedarfsgerechten Qualifizierung}

Um auf die zunehmende Verbreitung von Informations- und Kommunikationstechnologien in vielen Bereichen vorzubereiten und somit einer digitalen Spaltung entgegenzuwirken adressiert das Projekt FachWerk das Ziel, die Digitalisierung und Kompetenzentwicklung im Handwerk, als eine Branche, die bisher noch wenig an dem Megatrend partizipiert, voranzubringen. Hierfür entwickelte FachWerk ein multimediales Lehr- und Lernarrangement, das zur Fachkräftequalifizierung dient und auf die zukünftigen Herausforderungen beim Umgang mit IuK-Technologien vorbereiten soll. Letztlich kann so sichergestellt werden, dass das Handwerk als eine traditionsreiche und manuell geprägte Branche von den Vorteilen der Digitalisierung profitiert und auch

P. Spieth $\cdot$ C. Klos $\cdot$ T. Röth $(\square)$

Universität Kassel, Institut für Betriebswirtschaftslehre/Fachgebiet Technologie- und Innovationsmanagement sowie Entrepreneurship, Kassel, Deutschland

L. Schmidt · J. Funk · A. Klingauf

Universität Kassel, Institut für Arbeitswissenschaft und Prozessmanagement/Fachgebiet

Mensch-Maschine-Systemtechnik, Kassel, Deutschland

S. Robak $\cdot$ M. Knaut $\cdot$ M. Klimpel $\cdot$ L. Heidemann

Leibniz Universität Hannover, Institut für Berufspädagogik und Erwachsenenbildung/

Professur für Bildung im Erwachsenenalter, Hannover, Deutschland

F. Schüttler

Berufsförderungswerk des Handwerks gGmbH, Korbach, Deutschland

H. Gringel

Gringel Bau + Plan GmbH, Schwalmstadt, Deutschland

(C) Der/die Autor(en) 2021

W. Bauer et al. (Hrsg.), Arbeit in der digitalisierten Welt,

https://doi.org/10.1007/978-3-662-62215-5_4 
zukünftig erfolgreich in der Wirtschaft mitwirken kann. Um das Projektziel zu erreichen, wurden sieben Arbeitspakete definiert: (Abb. 4.1)

Im Rahmen des ersten Arbeitspakets (AP) wurde der Ist- sowie der Soll-Stand des Einsatzes von IuK-Technologien definiert und somit eine technologische Vorausschau erstellt. Hierzu führte das Fg TIME Experteninterviews sowie mehrere Workshops durch. Durch die Bereitstellung von Alternativ- und Zukunftsszenarien konnte außerdem eine strategische Vorausschau auf den Einsatz digitaler Technologien gegeben werden. Unterstützend analysierte das Fg MMS die Gebrauchstauglichkeit der IuK-Technologien für das Handwerk. Parallel zu AP 1 wurde im Rahmen des zweiten APs eine Bedarfsund Anforderungsanalyse aus Belegschaftssicht durchgeführt. Hierzu sammelte das BFH gemeinsam mit der Agentur für Arbeit über fast zwei Jahre Stellenanzeigen, um regionale Arbeitsplatzanforderungen darzustellen. Die Stichprobe mit einem Umfang von 352 Anzeigen wurde anschließend vom IfBE analysiert. Zusätzlich führte das IfBE eine Dokumentenanalyse sowie Leitfadeninterviews durch. Im Rahmen des dritten APs konnten fünf Erfolgsfaktoren identifiziert werden, welche die wichtigsten Ansatzpunkte zur digitalen Transformation festlegen. Hierfür analysierten die Praxispartner gemeinsam

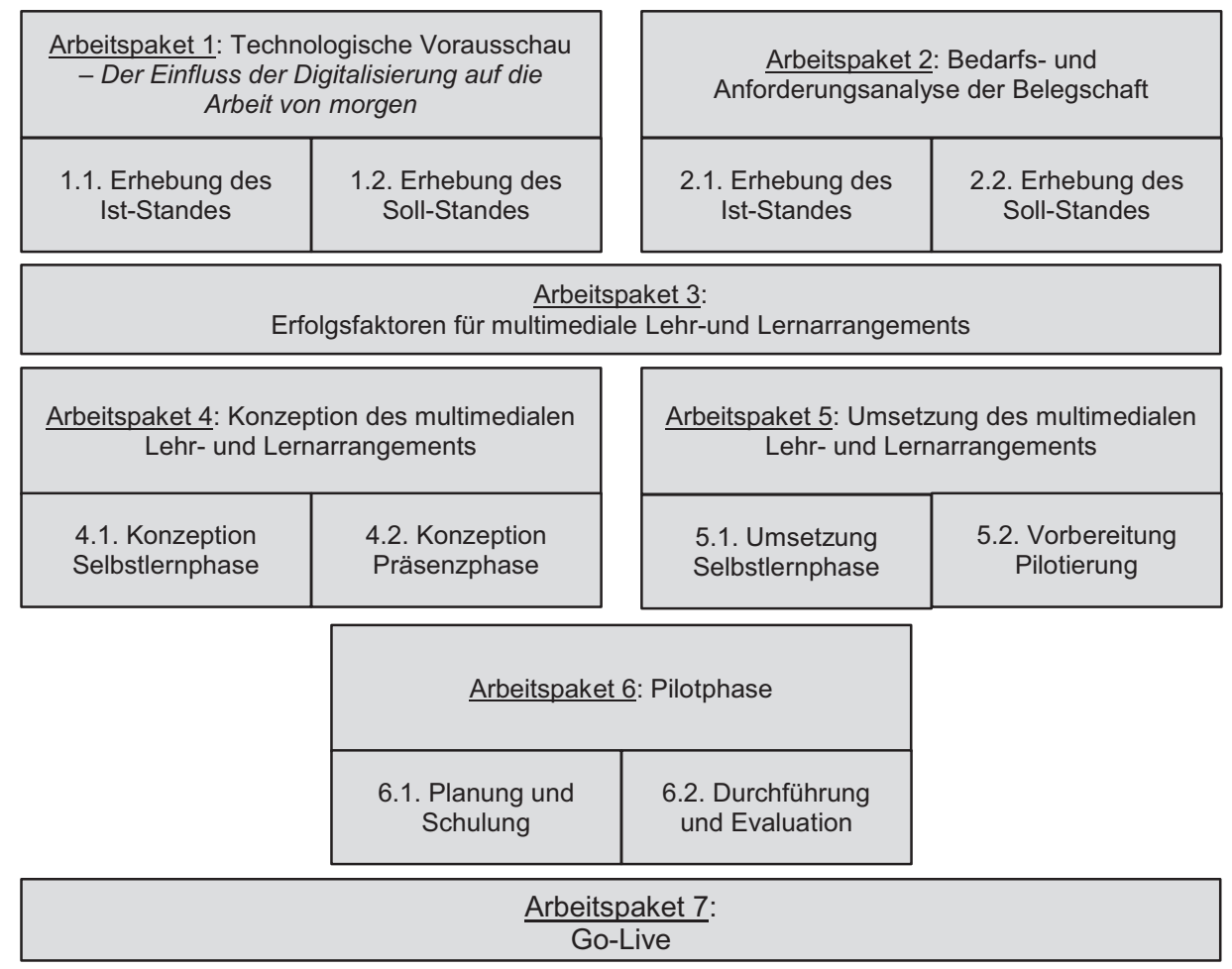

Abb. 4.1 Die Arbeitspakete des Projekts FachWerk 
mit dem Fg TIME die neuen Notwendigkeiten, identifizierten potenzielle Einsatzgebiete und nutzten die zuvor gewonnenen Workshop-Daten Interviews.

AP 4 diente zur Konzeption des multimedialen Lehr- und Lernarrangements. Hierfür entwickelte das IfBE in einem nachfrage-/bedarfsorientierten Prozess und unter Einbeziehung der Ergebnisse der AP 2 und 3 ein didaktisches Konzept. Basierend auf diesem Konzept und unter Einbeziehung der entwickelten Tätigkeitsprofile konnten didaktische Prinzipien identifiziert werden, die aus pädagogischer Sicht wesentliche Kriterien für die Gestaltung und Umsetzung darstellen. Darauf basierend entwickelte das BFH erste Schulungsinhalte und verknüpfte thematisch passende Inhalte zu übergreifenden Lerneinheiten. Im Rahmen des fünften APs wurde die multimediale Lehrund Lernplattform umgesetzt. Das sechste AP umfasste mehrere vom BFH durchgeführte Schulungen, die zur Pilotierung des multimedialen Lehr- und Lernarrangements dienten. Abschließend hat sich das siebte AP mit der regionalen und überregionalen Vernetzung sowie mit der abschließenden Evaluation durch das BFH befasst.

\subsubsection{Das Handwerk durch die Integration moderner Technologien digital transformieren}

Das Fg TIME definierte in einem ersten Schritt verschiedene Mega- und Technologietrends, die aktuell und zukünftig einen Einfluss auf die Handwerksbranche ausüben [15]. Megatrends bestimmen in diesem Zusammenhang Aspekte, die einen umfassenden Wandel für Gesellschaft und Wirtschaft mit sich bringen. Technologietrends hingegen gestalten den technologischen Wandel und bestimmen somit die Digitalisierung der Handwerksbranche [16]. Die identifizierten Megatrends umfassten Klimawandel und ökologische Nachhaltigkeit, Digitalisierung und New Work sowie Individualisierung und Globalisierung. So gewinnt beispielsweise die Fokussierung auf nachhaltiges Konsumverhalten und der Umbruch der Arbeitswelt für Unternehmen an Bedeutung. Technologietrends umfassten mobile Endgeräte und 3D-Druck, Einsatz neuer Medien, Cloud-Computing, Virtual Reality und das Internet der Dinge. Durch den Einsatz dieser Technologien können beispielsweise Erreichbarkeit und Flexibilität verbessert werden, neue Marktpotenziale ausgeschöpft und Planungs- und Entscheidungsprozesse beschleunigt werden [2]. Außerdem besteht die Möglichkeit, interaktive Wirklichkeiten zu gestalten und somit die Wahrnehmungserlebnisse von Handwerkerinnen und Handwerkern sowie Kunden zu verbessern. Auch Prozesse und Prozessteuerungen können durch die Vernetzung von Objekten optimiert und bedarfsgerechter gestaltet werden [10].

Neben den Mega- und Technologietrends hat das Fg TIME durch mehr als 80 Experteninterviews fünf Erfolgsfaktoren identifiziert, die die neuen und zukünftigen Anforderungen der Handwerksbranche durch die Implementierung digitaler Technologien umfassen: 
1. Relevanz der (hierarchie-) übergreifenden digitalen internen Kommunikation und Dokumentation. Digitale Technologien müssen genutzt werden, um Kommunikation flexibler und schneller zu gestalten.

2. Unterstützungsfunktion für nicht-digitalisierbare manuelle Arbeiten. Dies umfasst den Einsatz von Augmented-Reality Systemen und mobilen Anwendungen.

3. Ausschöpfung des Digitalisierungspotenzials für den Kundenkontakt. So können beispielsweise digitale Technologien neue Vertriebs- und Vernetzungsmöglichkeiten schaffen oder Produktpräsentationen und -dokumentationen verbessern.

4. Gewährleistung der Datensicherheit. Dabei spielen vor allem rechtliche Anforderungen und Vorgaben eine entscheidende Rolle sowie auch die Erfüllung der Stakeholdererwartungen.

5. Relevanz der bedarfsgerechten Gestaltung von internen und externen Schnittstellen. Die Adaptierfähigkeit und Kompatibilität von Systemen durch den Einsatz digitaler Technologien nimmt hierbei eine entscheidende Rolle ein.

Neben den identifizierten Mega- und Technologietrends sowie den definierten Erfolgsfaktoren konnte das Fg TIME über die gesamte Projektlaufzeit hinweg eine 2-jährige Studie durchführen, welche als Basis für die Entwicklung einer Messskala diente. Dabei kann die Messskala genutzt werden, um Aussagen über den technologischen Frame eines Individuums zu treffen, welcher die erste kognitive Reaktion beschreibt, die ein Mensch beim Kontakt mit digitalen Technologien erfährt [5]. Durch den Einsatz der Messskala im Handwerk kann beurteilt werden, wie Mitarbeitende auf die Implementierung neuer digitaler Technologien reagieren. Somit besteht die Möglichkeit, bedarfsgerechte Aus- und Weiterbildungen zu gestalten und beispielsweise die multimediale Lehr- und Lernplattform zu nutzen, um Mitarbeitende mit weniger positiven Reaktion auf digitale Technologien entsprechend zu schulen. Die Unterteilung der Messskala ermöglicht es, eine detaillierte Analyse des Meinungsbildes basierend auf (1) persönlicher Einstellung, (2) persönlicher Anwendung, (3) organisationalem Einfluss, (4) industriellem Einfluss und (5) Einfluss des Vorgesetzten durchzuführen. Um darauf basierend Aussagen über den technologischen Frame eines Individuums treffen zu können, wird eine Skala von 1 (trifft überhaupt nicht zu) bis 7 (trifft voll und ganz zu) verwendet. Im Folgenden wird die Skala zur Bewertung des technologischen Frames präsentiert: (Tab. 4.1) 
Tab. 4.1 Messskala zur Bewertung des technologischen Frames (eigene Darstellung)

\begin{tabular}{|c|c|c|c|c|c|c|c|c|}
\hline \# & Item & 1 & 2 & 3 & 4 & 5 & 6 & 7 \\
\hline $1 \mathrm{~A}$ & $\begin{array}{l}\text { Meine Einstellung gegenüber digitalen Technologien ist } \\
\text { positiv }\end{array}$ & & & & & & & \\
\hline $1 \mathrm{~B}$ & Ich habe hohe Erwartungen an digitale Technologien & & & & & & & \\
\hline $1 \mathrm{C}$ & Digitale Technologien sind ein wichtiger Teil meines Lebens & & & & & & & \\
\hline $1 \mathrm{D}$ & $\begin{array}{l}\text { Ich versuche regelmäßig Informationen über digitale } \\
\text { Technologien zu erhalten }\end{array}$ & & & & & & & \\
\hline $2 \mathrm{~A}$ & $\begin{array}{l}\text { Digitale Technologien können die Koordination meiner } \\
\text { Arbeitsaufgaben erleichtern }\end{array}$ & & & & & & & \\
\hline 2B & Digitale Technologien machen meine Arbeit flexibler & & & & & & & \\
\hline $2 \mathrm{C}$ & $\begin{array}{l}\text { Digitale Technologien verringern die Möglichkeit von } \\
\text { Fehlern bei der Arbeit }\end{array}$ & & & & & & & \\
\hline $2 \mathrm{D}$ & $\begin{array}{l}\text { Digitale Technologien erhöhen die Effektivität meiner } \\
\text { Arbeitsschritte }\end{array}$ & & & & & & & \\
\hline $3 \mathrm{~A}$ & $\begin{array}{l}\text { Meine Kollegen erinnern mich daran, digitale Technologien } \\
\text { im Arbeitsalltag zu nutzen }\end{array}$ & & & & & & & \\
\hline $3 B$ & $\begin{array}{l}\text { Meine Kollegen empfehlen mir regelmäßig digitale Techno- } \\
\text { logien }\end{array}$ & & & & & & & \\
\hline $3 \mathrm{C}$ & $\begin{array}{l}\text { Meine Kollegen erwarten dein Einsatz digitaler Techno- } \\
\text { logien im Job }\end{array}$ & & & & & & & \\
\hline $3 \mathrm{D}$ & $\begin{array}{l}\text { Meine Kollegen helfen mir digitale Technologien im Job zu } \\
\text { nutzen }\end{array}$ & & & & & & & \\
\hline $4 \mathrm{~A}$ & $\begin{array}{l}\text { Unsere Konkurrenten erwarten die Nutzung digitaler } \\
\text { Technologien }\end{array}$ & & & & & & & \\
\hline $4 B$ & $\begin{array}{l}\text { Unsere Konkurrenten nutzen digitale Technologien erfolg- } \\
\text { reich }\end{array}$ & & & & & & & \\
\hline $4 \mathrm{C}$ & Unsere Kunden erwarten die Nutzung digitaler Technologien & & & & & & & \\
\hline $4 \mathrm{D}$ & $\begin{array}{l}\text { Unsere Zulieferer erwarten die Nutzung digitaler Techno- } \\
\text { logien }\end{array}$ & & & & & & & \\
\hline $5 \mathrm{~A}$ & $\begin{array}{l}\text { Mein Vorgesetzter ist bereit, digitale Technologien in das } \\
\text { Unternehmen zu integrieren }\end{array}$ & & & & & & & \\
\hline $5 \mathrm{~B}$ & $\begin{array}{l}\text { Mein Vorgesetzter erwartet von mir den Einsatz digitaler } \\
\text { Technologien }\end{array}$ & & & & & & & \\
\hline $5 \mathrm{C}$ & $\begin{array}{l}\text { Mein Vorgesetzter spricht regelmäßig über digitale Techno- } \\
\text { logien }\end{array}$ & & & & & & & \\
\hline $5 \mathrm{D}$ & $\begin{array}{l}\text { Mein Vorgesetzter ist ein Experte im Umgang mit digitalen } \\
\text { Technologien }\end{array}$ & & & & & & & \\
\hline
\end{tabular}




\subsubsection{D-360'-Lerneinheit in der praktischen Ausbildung von Handwerkern}

In die Lernumgebung wurden verschiedene Werkzeuge für innovative Lernformen integriert, die vom Fachgebiet Mensch-Maschine-Systemtechnik der Universität Kassel als Unterstützung für die Lehrenden bereitgestellt wurden. Hierzu wurden Gamificationund Learning-Analytics-Plug-Ins ergänzt und eigene Werkzeuge zur Erstellung und Nutzung von Virtual-Reality-(VR)- und Augmented-Reality-(AR)-Inhalten entwickelt. Dabei wurde die Verknüpfung von digitalen Inhalten, beispielsweise auf der Lernplattform, mit Objekten und Orten in der realen Umgebung ermöglicht. Hierzu wurde u. A. die Software QR-Code-Helper als Werkzeug entwickelt. Außerdem wurden 3D-360Videos als leichter Einstieg in die Erstellung von VR-Inhalten untersucht und vorgestellt. Im Gegensatz zu aufwendig computergenerierten virtuellen Umgebungen können 3D-360'-Videos mit einer speziellen Kamera in der realen Umgebung verhältnismäßig einfach aufgezeichnet werden.

In einer Studie wurde untersucht, inwieweit 3D-360'-Videos als Lernmaterial geeignet sind. Hierzu wurden aktuell in der Ausbildung verwendete Lernvideos als Ausgangslage verwendet, die aus dem Forschungsprojekt FAINLAB stammten, in dem ein multimediales Lernangebot für die Ausbildung in der Bauwirtschaft erstellt wurde [10]. Als Untersuchungsgegenstand der Studie wurde das Modul „Herstellen eines Küchenfliesenspiegels“ als Arbeitsaufgabe ausgewählt. Dieser Arbeitsvorgang wurde in vier aufeinander aufbauende Schritte unterteilt. Dann wurde ein 3D-360 ${ }^{\circ}$ Video mit einem Auszubildenden, der sich in einem fortgeschrittenen Lehrjahr befand und geübt im Anfertigen eines Fliesenspiegels war, in einer Halle der Lehrbaustelle des Berufsförderungswerks des Handwerks aufgezeichnet [2]. Vier Arbeitsplätze wurden dabei mit je $90^{\circ}$ Versatz so um die $3 \mathrm{D}-360^{\circ}$-Kamera angeordnet, dass in jeder Blickrichtung jeweils ein Arbeitsschritt $\mathrm{zu}$ sehen ist. Der Auszubildende führte die vier Schritte direkt nacheinander an den jeweils dafür vorbereiteten Arbeitsplätzen durch. Mit der zusammenhängenden Aufzeichnung können Nutzer*innen dem Akteur beim Ausführen der Arbeitsaufgabe leicht folgen, sodass sie auf einfache und natürliche Art durch das 3D-360-Video geführt werden. Anschließend wurden Banner zur Markierung der einzelnen Schritte und Schaltflächen als Interaktionsmöglichkeit ergänzt, die beim Ansehen auf einem Smartphone-basierten Head-Mounted Display durch Kopfbewegungen angesteuert werden können. Wenn die Nutzer*innen eine Markierung in der Mitte des Sichtfeldes auf eine Schaltfläche ausrichten, löst nach 2,5 s die Funktion der Schaltfläche aus, was auch als Blicksteuerung bezeichnet wird. Die Verwendung dieser Eingabemethode wurde gewählt, da keine zusätzliche Hardware benötigt wird. Über ein Videomenü zentral unterhalb der Nutzer*innen können übliche Aktionen wie das Starten oder Pausieren des Videos gesteuert werden. Durch die gewählte Platzierung befindet sich das Menü während der Betrachtung der Arbeitsschritte außerhalb des Sichtfelds der Nutzer*innen und ist trotzdem jederzeit leicht erreichbar. 


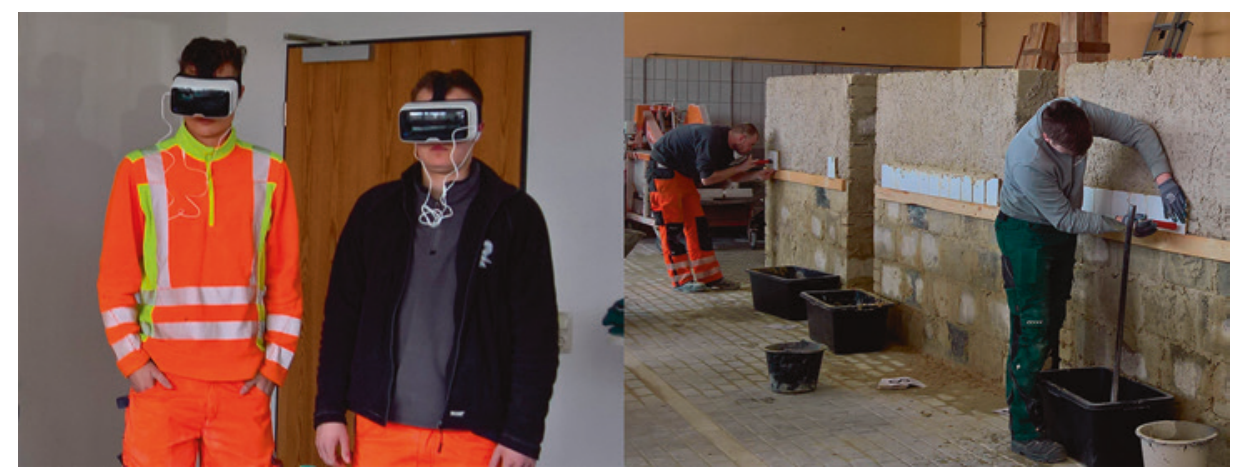

Abb. 4.2 Zwei Nutzer der 3D-360-Lerneinheit zum Anfertigen eines Fliesenspiegels mit einem smartphone-basiertem Head-Mounted Display (links) und Auszubildende bei der praktischen Umsetzung des zuvor Gelernten in der Halle der Lehrbaustelle (rechts)

Die erstellte Anwendung wurde in einer Studie mit Auszubildenden zum Tief- bzw. Hochbaufacharbeiter im ersten Lehrjahr $(n=20)$ mit der konventionellen Lerneinheit verglichen [7]. Abb. 4.2 zeigt je zwei Teilnehmer der Studie beim Lernen mit der 3D-360'-Anwendung sowie bei der praktischen Umsetzung des Gelernten. Es konnten keine signifikanten Unterschiede beim Lernerfolg festgestellt werden; beide Gruppen erreichten beim praktischen Fliesenlegen hohe durchschnittliche Werte im obersten Viertel der Skala. Es konnte außerdem gezeigt werden, dass die Motivation in der Gruppe mit 3D-360-Video signifikant höher war als in der Gruppe mit der konventionellen Lerneinheit. Auch die subjektive Meinung der Lernenden zu der genutzten Technologie war in der $3 \mathrm{D}-360^{\circ}$-Gruppe signifikant besser. Somit ergibt sich für Ausbildungsbetriebe die Chance, ihre Attraktivität mit dem Einsatz von 3D-360-Lernmethoden zu steigern, ohne dabei den Lernerfolg zu gefährden. Die erstellte Anwendung kann helfen, die nötige Lernmotivation zu gewährleisten und so zur Lösung einer wichtigen Herausforderung der Lehre beitragen [13].

Die für die Studie erstellte Anwendung gewann im AVRiL-Wettbewerbs 2019 für „Gelungene VR/AR-Lernszenarien“ der Fachgruppen Bildungstechnologien und VR/ AR der Gesellschaft für Informatik (GI) in Zusammenarbeit mit dem Stifterverband eine Sonderauszeichnung in der Kategorie „Interaktive $360^{\circ}$-Videos“. Hierbei wurde die Nutzung einfacher Techniken, mit denen sich schnell neue Inhalte aufbauen lassen, als Vorteil der Anwendung gelobt. Weiterhin wurde die Studie als „Projekt des Monats“ im Dezember 2019 auf der Webseite des GI-Arbeitskreises "VR/AR-Learning" vorgestellt [1]. Die Studie und deren Ergebnisse wurden zusätzlich auf der LEARNTEC 2020 in der VR/AR-Area präsentiert. 


\subsubsection{Bedarfsanalyse aus Fachkräftesicht sowie Konzeption und Erprobung des Lernarrangements}

Die Universität Hannover hat eine umfassende Bedarfs- und Anforderungsanalyse aus Fachkräftesicht durchgeführt sowie das didaktische Konzept entwickelt. Aus der multimethodischen, komplexen Bedarfs- und Anforderungsanalyse aus Fachkräftesicht konnten zwei detaillierte Tätigkeitsprofile der vorab definierten Zielgruppe der Mitarbeitenden der Praxispartner (Bau+Elektro) abgeleitet werden. Durch schrittweise Zusammenführung und Abstraktion erlauben die Profile eine Rekonstruktion aller Tätigkeiten auf selbst niedrigster Abstraktionsebene und zeigen so das konkrete Potenzial für den Einsatz digitaler Technologien auf jeder Tätigkeitsebene. Außerdem ist Verallgemeinerung durch abstrahierende Zusammenfassung auf höherer Ebene möglich. Die Tätigkeitsprofile stellen die Basis zur Identifikation von Anforderungen und Bedarfen dar. Die Tätigkeitsprofile orientieren sich an vier Kompetenzbereichen (Fach-, Sozial-, Selbst- und Medienkompetenz), denen die Tätigkeitsbereiche zugeordnet sind. Die tabellarisch vorliegenden Tätigkeitsprofile sind basierend auf einem mehrstufigen Reflexionsprozess zur Validierung der detaillierten Tätigkeitsstrukturen und prognostischer Darstellungen entstanden. In diesem Rahmen erfolgte außerdem auch eine Priorisierung der prognostizierten Veränderungen der Tätigkeiten nach Relevanz durch die Unternehmen (Kriterien: praktische Relevanz/zeitnahe Umsetzbarkeit i. d. nächsten 5 Jahren). Die Zusammenführung der Mitarbeitenden- und Unternehmenssicht verdeutlicht zentrale Anknüpfungspunkte für den Umgang mit IuK-Technologien im

Tab. 4.2 Bedürfnisse und Bedarfe im Kontext der Nutzung von IuK-Technologien im Handwerk (eigene Darstellung)

\begin{tabular}{|c|c|}
\hline Subjektive Bedürfnisse/Bedarfe & Objektive Bedarfe \\
\hline $\begin{array}{l}\text { Digitalisierung vor- und nachgelagerter } \\
\text { Röutinetätigkeiten } \\
\text { - Dokumentation } \\
\text { - Messung } \\
\text { - Datenauslese }\end{array}$ & $\begin{array}{l}\text { Digitalisiertes Dokumentenmanagement } \\
\text { - Verwendung digitaler Dokumente } \\
\text { - Erstellung/Bearbeitung digitaler Dokumente }\end{array}$ \\
\hline $\begin{array}{l}\text { Beschaffung relevanter Informationen } \\
\text { - Normen } \\
\text { - Bedienungsanleitungen }\end{array}$ & $\begin{array}{l}\text { Nutzung zentraler Speicherorte für digitale } \\
\text { Dokumente } \\
\text { - Server/Cloud } \\
\text { - Offline Speicherung auf Endgeräten }\end{array}$ \\
\hline $\begin{array}{l}\text { Digitalisierung der Arbeitsorganisation } \\
\text { - Zeiterfassung } \\
\text { - Planungsunterlagen } \\
\text { - Kollegiale Absprachen }\end{array}$ & $\begin{array}{l}\text { Digitalisierung der Arbeitsorganisation } \\
\text { - Online Dispositionsplan } \\
\text { - Liefer- und Bestellscheine } \\
\text { - Zeiterfassung }\end{array}$ \\
\hline $\begin{array}{l}\rightarrow \text { Zeitersparnis } \\
\rightarrow \text { Reduktion des Papierkonsums }\end{array}$ & $\begin{array}{l}\rightarrow \text { Dezentrale zugriffe auf relevante } \\
\text { Dokumente } \\
\rightarrow \text { Simplifizierte Dokumentenablage }\end{array}$ \\
\hline
\end{tabular}


Handwerk, aus denen sich ein digitales Entwicklungspotenzial und die Notwendigkeit einer entsprechenden Qualifizierung ergeben (Tab. 4.2.)

Die didaktische Konzeption des multimedialen Lehr- und Lernarrangements fokussiert die Kompetenzentwicklung zur Bewältigung von Anforderungen der Digitalisierung von Arbeit im Handwerk. Inhaltlich konnten basierend auf den identifizierten Bedarfen und erstellten Szenarien zusammenfassend und abstrahierend vier zentrale Themenfelder als wichtigste Schulungsinhalte bestimmt werden: 1. Server-/Cloudlösungen, 2. Digitales Dokumentenmanagement, 3. Mediennutzung/Medienkritik, 4. Datenschutzrecht/Datensicherheit. Zudem wurden fünf übergeordnete didaktische Prinzipien herausgearbeitet, die aus pädagogischer Sicht wesentliche Kriterien für die Gestaltung und Umsetzung des multimedialen Lehr- und Lernarrangements zur Qualifizierung für eine zukünftige Nutzung digitaler Technologien im Handwerk darstellen: 1. Handlungsorientierung, 2. Beziehungsorientierung, 3. Tätigkeitsbezogene Zielgruppenorientierung, 4. Selbstlernorientierung, 5. Praxisorientierung. Abgeleitet wurden konkrete Handlungsempfehlungen, welche den aktuellen Diskurs fundiert erweitern und konkretisieren.

\subsubsection{Bedarfsgerechte Qualifizierung im Handwerk: Entwicklung und Erprobung des digitalen Lehr-Lernarrangements}

Basierend auf den geleisteten Tätigkeiten wurden durch das Berufsförderungswerk des Handwerks Schulungsinhalte in Form von Lernzielen vordefiniert und anschließend erstellt. Die Festlegung der Inhalte erfolgte unter kontinuierlichem Austausch mit der Geschäftsführung des Projektbetriebs aus der Baubranche. Parallel dazu wurden die entwickelten Schulungsinhalte kontinuierlich mit dem didaktischen Konzept, welches im Rahmen des Projekts erstellt wurde, abgeglichen. Abschließend wurden die Schulungsunterlagen final erarbeitet. Um konkrete Rahmenbedingungen zu schaffen, einigten sich alle Projektteilnehmenden im Vorhinein darauf, zwei Schulungen mit unterschiedlichen Themen durchzuführen. Jede Schulung sollte insgesamt 18 Unterrichtseinheiten (UE) umfassen, die sich wie folgt aufteilen: 8 UE Präsenzunterricht, 6 UE Selbstlernphase über die zu entwickelnde Lernplattform als web-based Training und 4 UE im betrieblichen Kontext. Um trotz der durchzuführenden Schulungen keinen Zeitdruck im Arbeitsalltag zu verursachen, einigte man sich konkret auf folgenden Zeitplan für jede einzelne Schulung: (Abb. 4.3)

\begin{tabular}{|c|c|c|c|}
\hline $\begin{array}{c}\text { Präsenzphase 1 } \\
\text { Kick-Off Veranstaltung } \\
\text { (Woche 1) }\end{array}$ & \multicolumn{2}{|c|}{$\begin{array}{c}\text { Selbstlernphase 1 } \\
\text { (Woche 1-3) }\end{array}$} & \multicolumn{2}{|c|}{$\begin{array}{c}\text { Präsenzphase 2 } \\
\text { (Woche 4) }\end{array}$} \\
\hline $\begin{array}{c}\text { Selbstlernphase 2 } \\
\text { (Woche 4-7) }\end{array}$ & $\begin{array}{c}\text { Online- } \\
\text { Präsenztreffen 1 } \\
\text { (Woche 7) }\end{array}$ & $\begin{array}{c}\text { Praxis-Transfer- } \\
\text { Phase } \\
\text { (Woche 7-11) }\end{array}$ & $\begin{array}{c}\text { Online- } \\
\text { Präsenztreffen 2 } \\
\text { (Woche 12) }\end{array}$ \\
\hline
\end{tabular}

Abb. 4.3 Zeitplan zur Durchführung der Schulungen im Rahmen des Projekts 
Außerdem wurden die konkreten Inhalte der geplanten Schulungen endgültig festgelegt. Dabei wurde für Schulung 1 die thematische Auseinandersetzung mit Mediennutzung und Medienkritik sowie Datenschutzrecht und Datensicherheit festgelegt. Als Inhalte für die zweite Schulung wurden die Themen Server-/Cloudlösungen und digitales Dokumentenmanagement vorgesehen. Parallel wurden die technischen Voraussetzungen und verschiedenen Optionen der Lernplattform festgelegt und in ihrer Relevanz bewertet. Somit wurde sichergestellt, dass alle technischen Möglichkeiten zur Steigerung der Lernmotivation und des Lernerfolgs für die Schulungsteilnehmenden durch die Autoren ausgeschöpft werden können. Alle erstellten Schulungsinhalte wurden zunächst mit Teilnehmenden an diversen Weiterbildungsmaßnahmen beim Berufsförderungswerk des Handwerks getestet.

\subsection{Die Digitalisierung stellt das Handwerk vor Herausforderungen}

Viele Handwerkerinnen und Handwerker empfinden den digitalen Transformationsprozess als komplex und zeitaufwendig. Insbesondere System- und Medienbrüche verhindern oft die digitale Transformation einzelne Prozessschritte in KMUs [16]. Aus Sicht des Projekts FachWerk ergeben sich folgende Herausforderungen für das deutsche Handwerk in der digitalen Zukunft:

- Die einfache Integration digitaler Technologien reicht in den meisten Fällen nicht aus um einen tatsächlichen Mehrwert zu generieren [7]. Aus diesem Grund sollten digitale Geschäftsmodellinnovationen entwickelt werden, welche es auch kleinen Handwerksbetrieben ermöglichen, auf Plattformen und in Ecosystemen zu agieren [16]. Insbesondere die Verknüpfung zwischen den KMUs des Handwerks und der fertigenden Industrie sollte im Mittelpunkt der zukünftigen Branchenentwicklung stehen.

- Während nur die Hälfte der gering qualifizierten Beschäftigten beruflich IKT nutzt, sind es unter den Hochqualifizierten fast alle. Die Beschäftigten erfahren durch die digitale Transformation einerseits körperliche Erleichterung, andererseits sehen aber auch etwa $80 \%$ der Mitarbeitenden die Notwendigkeit sich weiterzuentwickeln und die eigenen Kompetenzen zu erhöhen [2]. Aus diesem Grund besteht eine Angebotslücke an innovativen und digitalen Schulungsformaten, welche die Mitarbeitenden im Handwerk bei der Kompetenzentwicklung unterstützen und so die digitale Transformation vorantreiben [8].

- Leider mangelt es an Studien, welche die Beschäftigten selbst und ihre individuelle Sicht und Wahrnehmung in den Vordergrund stellen [13]. Der Zentralverband des deutschen Handwerks hat hierzu treffend festgestellt: „Je kleiner der Handwerksbetrieb, desto größer der Bedarf für Sensibilisierungs- und Beratungsmaßnahmen rund um das Thema IT-Sicherheit und desto größer die Wahrscheinlichkeit, durch ITSicherheitslücken wirtschaftliche Schäden zu erleiden.“ [18]. Im Projekt FachWerk 
haben wir ähnliche Erfahrungen mit Mitarbeitenden der Handwerksbranche gemacht und empfehlen das Thema Daten- und IT-Sicherheit weiter in den Fokus der digitalen Transformation des Handwerks zu stellen [3].

\subsection{Weiterführende Literatur}

Eine ausführliche Zusammenfassung zu unseren Workshops können Sie der ersten FachWerk-Broschüre entnehmen (kostenfreier Download-Link):

https://www.upress.uni-kassel.de/katalog/abstract.php?978-3-7376-0560-1

Die Zusammenfassung der Projektarbeiten entnehmen Sie bitte der zweiten FachWerkBroschüre (kostenfreier Download-Link):

https://kobra.uni-kassel.de/handle/123456789/11468

\section{Projektpartner und Aufgaben}

- Fachgebiet Technologie- und Innovationsmanagement sowie Entrepreneurship der Universität Kassel [Fg TIME]

Vorausschau und Bedarfsanalyse zur Entwicklung der Plattform

Analysen im Innovations- und Technologiebereich zur Entwicklung des Lernarrangements

- Fachgebiet Mensch-Maschine-Systemtechnik der Universität Kassel [Fg MMS]

Teilvorhaben: Vorausschau und Bedarfsanalyse zur Entwicklung einer Lernplattform sowie deren Evaluation:

Analysen von Systemen, die das Zusammenwirken von Menschen mit Technik optimieren

- Institut für Berufspädagogik und Erwachsenenbildung der Universität Hannover [IfBE]

Teilvorhaben: Bedarfsanalyse aus Fachkräftesicht, Konzeption und Erprobung des Lernarrangements

Thematisierung von zentralen Fragen der Aus- und Weiterbildung sowie der Erwachsenenbildung

- Berufsförderungswerk des Handwerks gGmbH [BFH]

Teilvorhaben: Bedarfsgerechte Qualifizierung im Handwerk Entwicklung und Erprobung des Lehr-Lernarrangements:

Inhaltserarbeitung der Plattform und Durchführung von Lehrgängen sowie Sicherstellung der Verwertbarkeit

- Gringel Bau + Plan GmbH

Teilvorhaben: Digitalisierung im Bauhandwerk Erprobung und Evaluation sowie Fachkräftequalifizierung

Praxispartner, der die Sicherstellung der Praktikabilität sowie die bedarfsorientierte Ausrichtung der Projektinhalte kontinuierlich sicherstellt 


\section{- Agentur für Arbeit Korbach (assoziiert)}

Unterstützungsfunktion über die gesamte Projektlaufzeit zur verbesserten Qualifikation von erwerbslosen Fachkräften

- Handwerkskammer Kassel (assoziiert)

Teilnahme und Durchführung von Workshops, um den Praxisbezug sicherzustellen

\section{Literatur}

1. Arbeitskreis der GI-Fachgruppen Bildungstechnologien \& VR/AR: Einsatz einer 3D-360'-Lerneinheit in der praktischen Ausbildung von Handwerkern. https://www.uni-potsdam.de/vrarl/index.php/2019/12/02/einsatz-einer-3d-360-lerneinheit-in-der-praktischenausbildung-von-handwerkern/. Zugegriffen: 19. Febr 2020

2. BMAS (2016) Monitor: Digitalisierung am Arbeitsplatz. https://www.bmas.de/SharedDocs/ Downloads/DE/PDF-Publikationen/a875-monitor-digitalisierung-am-arbeitsplatz.pdf? blob=publicationFile \&v=2 Zugegriffen: 24. Febr 2020

3. BSI \& ZDH (2019) BSI und ZDH stellen IT-Grundschutzprofil für Handwerksbetriebe vor. https://www.bsi.bund.de/DE/Presse/Pressemitteilungen/Presse2019/GS-Profil-Handwerk_280319.html Zugegriffen: 24. Febr 2020

4. Colbert A, Yee N, George G (2016) The digital workforce and the workplace of the future. Acad Manag J 59:731-739. https://doi.org/10.5465/amj.2016.4003

5. Cornelissen JP, Werner MD (2014) Putting framing in perspective: a review of framing and frame analysis across the management and organizational literature. Acad Manage Ann 8:181235

6. Funk J, Klingauf A, Lüüs L Schmidt 2019 Umsetzung einer 3D-360-Lerneinheit in der praktischen Ausbildung von Handwerkern: Einsatzmöglichkeiten von interaktiven immersiven Medien S Schulz Hrsg ProceedinA, gs of DELFI workshops (Berlin 2019) Gesellschaft für Informatik Bonn 161172

7. Helfat CE, Raubitschek RS (2018) Dynamic and integrative capabilities for profiting from innovation in digital platform-based ecosystems. Res Policy 47:1-9. https://doi.org/10.1016/j. respol.2018.01.019

8. Hemerling J, Kilmann J, Danoesastro M, Stutts L, Ahern C (2018) It's not a digital transformation without a digital culture. Boston Consulting Group. https://www.bcg.com/ publications/2018/not-digital-transformation-without-digital-culture.aspx Zugegriffen 24. Febr 2020

9. Klingauf A, Funk J, Lüüs A, Schmidt L (2019) Wirkung von interaktiven 3D-360-Lernvideos in der praktischen Ausbildung von Handwerkern. In: Pinkwart N, Konert J (Hrsg) DELFI 2019: 17. Fachtagung Bildungstechnologien (Berlin 2019). Lecture Notes in Informatics (LNI) - Proceedings Bd. P-297. Gesellschaft für Informatik, Bonn, pp 145-156

10. Klos C, Spieth P (2019) Measuring the effects of technology framing: development of a multiple item scale. Acad Manag Proc 2019:13607. https://doi.org/10.5465/AMBPP.2019.229

11. Matt C, Hess T, Benlian A (2015) Digital transformation strategies. Bus Inf Syst Eng 57:339343. https://doi.org/10.1007/s12599-015-0401-5

12. Meyser J (2009) FAINLAB: Ein Projekt zur Förderung des multimedialen Lernens in der Ausbildung der Bauwirtschaft. Die berufsbildende Schule 61:115-121 
13. PwC (2018) Workforce of the future: the competing forces shaping 2030, pp 1-42

14. Riedl A, Schelten A (2013) Grundbegriffe der Pädagogik und Didaktik beruflicher Bildung. Steiner, Stuttgart

15. Rohrbeck R, Battistella C, Huizingh E (2015) Corporate foresight: An emerging field with a rich tradition. Technol Forecast Soc Chang 101:19. https://doi.org/10.1016/j. techfore.2015.11.002

16. Teece DJ (2018) Profiting from innovation in the digital economy: enabling technologies, standards, and licensing models in the wireless world. Res Policy 47:1367-1387. https://doi. org/10.1016/j.respol.2017.01.015

17. Welzbacher C, Pirk W, Ostheimer A, Bartelt K, Bille J, Klemmt M (2015) Digitalisierung der Wertschöpfungs-und Marktprozesse - Herausforderungen und Chancen für das Handwerk. Verein zur Förderung des Heinz-Piest-Instituts für Handwerkstechnik

18. ZDH (2020) 20 „IT-Sicherheitsbotschafter im Handwerk“ erhalten ihre Urkunden. https:// www.zdh.de/fachbereiche/zentralbereich/sicher-im-internet/it-sicherheitsbotschafter-im-handwerk. Zugegriffen: 24. Febr 2020

Open Access Dieses Kapitel wird unter der Creative Commons Namensnennung 4.0 International Lizenz (http://creativecommons.org/licenses/by/4.0/deed.de) veröffentlicht, welche die Nutzung, Vervielfältigung, Bearbeitung, Verbreitung und Wiedergabe in jeglichem Medium und Format erlaubt, sofern Sie den/die ursprünglichen Autor(en) und die Quelle ordnungsgemäß nennen, einen Link zur Creative Commons Lizenz beifügen und angeben, ob Änderungen vorgenommen wurden.

Die in diesem Kapitel enthaltenen Bilder und sonstiges Drittmaterial unterliegen ebenfalls der genannten Creative Commons Lizenz, sofern sich aus der Abbildungslegende nichts anderes ergibt. Sofern das betreffende Material nicht unter der genannten Creative Commons Lizenz steht und die betreffende Handlung nicht nach gesetzlichen Vorschriften erlaubt ist, ist für die oben aufgeführten Weiterverwendungen des Materials die Einwilligung des jeweiligen Rechteinhabers einzuholen.

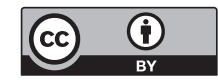

